\title{
Atividade Antimicrobiana de Extrato Bruto e Fração Hexânica de Alecrim - Rosmarinus officinalis L. (Lamiaceae), Frente à Patógenos Alimentares.
}

Luíza Toubas Chaul (I), Stone de Sá (I), Danillo Luiz dos Santos (I), Edemilson Cardoso da Conceição (I), Virgínia Farias Alves (I), José Realino de Paula (I)

(I) FF-UFG - Faculdade de Farmácia - Universidade Federal de Goiás (Goiânia, GO)

\section{Resumo}

INTRODUÇÃO: A segurança alimentar representa um grande desafio para a indústria alimentícia, especialmente porque nos últimos anos os consumidores tornaram-se mais exigentes e buscam alimentos de alta qualidade e livres de conservadores químicos. Essa realidade tem impulsionado a busca por alternativas naturais de conservação de alimentos. OBJETIVO: O presente estudo objetivou avaliar a atividade antimicrobiana do extrato bruto e da fração hexânica de Rosmarinus officinalis L. (Lamiaceae), popularmente conhecido como alecrim, frente a patógenos alimentares. PARTE EXPERIMENTAL: O material botânico foi obtido comercialmente de fornecedor local. As folhas, previamente secas, foram moídas em liquidificador industrial. Obteve-se o extrato bruto por percolação, concentrado em rotaevaporador e a fração hexânica foi obtida por partição. Em seguida, foram realizados testes de microdiluição em caldo, para a determinação da concentração inibitória mínima (CIM). Os patógenos testados foram Listeria monocytogenes ATCC 7644; Bacillus cereus ATCC 14579; Escherichia coli ATCC 8739 e Salmonella enterica subsp. enterica serovar Typhi ATCC 19430. RESULTADOS E DISCUSSÃO: O extrato bruto de R. officinalis apresentou fraca atividade $(\mathrm{CIM}=1000 \mu \mathrm{g} / \mathrm{mL})$ frente aos micro-organismos testados. Por outro lado, a fração hexânica apresentou boa atividade $(\mathrm{CIM}=125 \mu \mathrm{g} / \mathrm{mL})$ frente a $\mathrm{L}$.

\footnotetext{
Referência:

Luíza Toubas Chaul, Stone de Sá, Danillo Luiz dos Santos, Edemilson Cardoso da Conceição, Virgínia Farias Alves, José Realino de Paula. Atividade Antimicrobiana de Extrato Bruto e Fração Hexânica de Alecrim - Rosmarinus Officinalis L. (Lamiaceae), Frente À Patógenos Alimentares.. In: Anais do 12을 Congresso Latinoamericano de Microbiologia e Higiene de Alimentos - MICROAL 2014 [= Blucher Food Science Proceedings, num.1, vol.1]. São Paulo: Editora Blucher, 2014. 
monocytogenes ATCC 7644 e B. cereus ATCC 14579, atividade moderada frente a E. coli ATCC $8739(\mathrm{CIM}=250 \mu \mathrm{g} / \mathrm{mL})$ e S. enterica subsp. enterica serovar Typhi ATCC $19430(\mathrm{CIM}=500 \mu \mathrm{g} / \mathrm{mL})$. Estudos prévios observaram que a eficácia antimicrobiana de R. officinalis estava associada à sua composição fenólica, sendo que essa atividade era maior em frações do que no extrato bruto. A fração hexânica desse vegetal apresenta em sua constituição diterpenos fenólicos (ácido carnósico e carnosol), que possivelmente estão diretamente associados à sua atividade antimicrobiana. Os resultados obtidos no presente trabalho sugerem que a fração hexânica de R. officinalis apresenta potencial promissor na busca de alternativas naturais visando o controle de patógenos em alimentos.

Palavras-Chave: Antimicrobianos naturais, Rosmarinus officinalis, patógenos alimentares

Agência de Fomento: CAPES, CNPq (INCTTOX), FAPEG 